\title{
Search Probability for Non-zero Effects Detection under Skew-Normal/Independent Search Model
}

\author{
Sara Sadeghi and Hooshang Talebi* \\ University of Isfahan \\ Received: 5/23/2018 Approved: 12/31/2018
}

\begin{abstract}
Shirakura et al. (1996) has been introduced and calculated the search probability $(S P)$ for normal search model. However, in practical situations the normality assumption may fail. In this study, we consider a more realistic underlying skew-normal/independent $(S N I)$ model and obtain the $S P$. This is a general case, in a sense that the result in Shirakura et al. (1996) is its special case. The proposed $S P$ carries some reliable properties and can be used as a design comparison criterion to compare and rank the search designs $(S D)$.
\end{abstract}

Keywords. Design comparison criterion; search design; search linear model; search probability; skew-normal distribution.

MSC 2010: 62k15.

\section{Introduction}

Expensive treatments in many factorial designs rally the cost of experiments. The challenge for saving money is running the experiments with the lower number of runs. So, experimenters often apply a fraction of factorial experiment, assuming the higher order interactions are all negligible; e.g. in

\footnotetext{
${ }^{*}$ Corresponding author

Copyright $(\mathrm{C} 2019$, ASP Ins. This open-access article is published under the terms of the Creative Commons AttributionNonCommercial 4.0 International License which permits Share (copy and redistribute the material in any medium or format) and Adapt (remix, transform, and build upon the material) under the Attribution-NonCommercial terms.
} 
main effect plans $(M E P)$ it is assumed that all interaction effects are zero. Meanwhile, the existence of non-zero interactions may lead to the biased estimation of main effects, though the sparsity principle indicates that the number of such the interactions is small.

A search design resolves the search and estimation non-zero interaction effects along with estimating the overall mean and main effects. For $k$ nonzero interactions, such a $S D$ is called a main effect plus $k$ plan (MEP.k). Construction of $M E P . k$ has been investigated by several authors and were reviewed in Ghosh et al. (2007).

Following Srivastava et al. (1975), for an $N \times 1$ vector of observations $\mathbf{y}$, the search linear model is defined as

$$
\mathbf{y}=\mathbf{A}_{1} \boldsymbol{\xi}_{1}+\mathbf{A}_{2} \boldsymbol{\xi}_{2}+\mathbf{e}, \quad \operatorname{Var}(\mathbf{e})=\sigma^{2} \boldsymbol{\Sigma}_{\mathbf{e}}
$$

where $\mathbf{A}_{i}$ 's $(i=1,2)$ are known design $\left(N \times \nu_{i}\right)$ matrices and $\boldsymbol{\xi}_{i}$ 's $(i=1,2)$ are $\left(\nu_{i} \times 1\right)$ vectors of factorial effects for $i=1,2$, e is an $(N \times 1)$ error vector, $\sigma^{2}$ is unknown coefficient of covariance matrix $\boldsymbol{\Sigma}_{\mathbf{e}}$. It is known that $k$ elements of $\boldsymbol{\xi}_{2}$ are non-zero but do not know which ones they are. Meanwhile, $\boldsymbol{\xi}_{1}$ is the set of effects which the interest is to be estimated all along with identifying and estimating the non-zero effects in $\boldsymbol{\xi}_{2}$. A design which resolve such the problem is called a search design. For each vector of possible $k$ non-zero effects $\boldsymbol{\zeta}(k \times 1)$ in $\boldsymbol{\xi}_{2}$ let $\mathbf{A}_{21}(\boldsymbol{\zeta})$ be the $N \times k$ submatrix of $\mathbf{A}_{\mathbf{2}}$ corresponding to $\boldsymbol{\zeta}$. The emanated reduced models are written as below,

$$
\mathbf{y}=\mathbf{A}_{1} \boldsymbol{\xi}_{1}+\mathbf{A}_{21}(\boldsymbol{\zeta}) \boldsymbol{\zeta}+\mathbf{e}
$$

There are $l=\left(\begin{array}{c}\nu_{2} \\ k\end{array}\right)$ rival models in $(2)$, only a model is true, i.e.:

$$
\mathbf{y}=\mathbf{A}_{1} \boldsymbol{\xi}_{1}+\mathbf{A}_{21}\left(\boldsymbol{\zeta}_{0}\right) \boldsymbol{\zeta}_{0}+\mathbf{e}
$$

where $\boldsymbol{\zeta}_{0}$ is $k \times 1$ correct vector of non-zero effects in $\boldsymbol{\xi}_{2}$. The search problem is to identify and estimate $\boldsymbol{\zeta}_{0}$ as well as estimate $\boldsymbol{\xi}_{1}$. To identify $\boldsymbol{\zeta}_{0}$, Srivastava et al. (1975) suggested to calculate sum of square error $(S S E)$ for all possible $l$ models (2) and appointed the model with minimum $S S E$ as the true model. Shirakura et al. (1996) argued about the stochastic property of $S S E$ and proposed to initiate $P\left(S S E_{0} \leqslant S S E\right)$ on $S S E_{0}$ of model (3) and $S S E$ of any other competing model and came up with the search probability in an explicit form for $k=1$ and underlying normal search linear model. That is, the search probability is the minimum value of the probability of 
paired comparing of the $S S E$ s for all possible rival models. $S P$ is design dependent and thus, introduced as a measure for comparing $S D$ s with respect to their search performances. That is, the $S D$ with a higher $S P$ is superior in identifying the true model. Several $S P$-based criteria have been proposed by researchers for comparing search ability of $S D$ s. As examples, Ghosh and Teschmacher (2002) and Talebi and Esmailzadeh (2011b) developed the above $S P$-criterion, all are restricted to $k=1$. Talebi and Esmailzadeh (2011a) gave a so-called $K L$-criterion which is applicable for $k \geqslant 1$.

All of the above criteria including Shirakura et al. (1996) have been obtained for normal errors; however, in practical situations such an assumption may not be well adapted to real data. There are numerous number of non-normal real data set, e.g. with skewed or heavy tailed distributions. For example, hidden truncation problem which has been described by Arnold et al. (2002), was led to data with skew-normal $(S N)$ model; Data set in Bahrami and Qasemi (2015) were analyzed by a flexible skewgeneralized normal model; AIS data in Marchenko (2010) and wind speed data in Arellano-Valle and Genton (2010) were considered and analyzed as $S N$ and skew-t models; In analyzing fiber-glass data, Lachos and Labra (2014) applied distributions from SNI class. They developed SNI family distributions including models with skew-normal, skew-t, skew-slash and skew-contaminated normal distributions. Additionally, this family comprise the normal/independent distributions as a special case.

For the non-normal real data, the developed $S P$-based criteria are ineffective. So, in order to cover the real problem situations and due to practical flexibility and utility of the $S N I$ family, $S P$ for $S N I$ search models was studied in this research. This generalized the results of Shirakura et al. (1996), i.e. the $S P$ for normal model would be a special case of this study. So, the criterion obtained under the $S N I$ model is more general which allows for more flexibility in design comparisons.

Some preliminaries on distributional issues are required that are presented in Section 2. In Section 3, the formulization of $S P$ is given. Under $S N$ and $S N I$ error, the $S P$-criterion will be obtained and presented in Section 4. The performance of the proposed criterion in comparing the search ability and ranking of some $S D$ s are given in Section 5 . 


\section{Distributional Perspective}

Because in this research a general form of $S P$ for linear search models with $S N I$ error will be investigated, more understandings of $S N I$ and $S N$ distributions and some of features are helpful.

A random variable $Z$ is distributed as a $S N$ distribution with skewness parameter $\lambda$, denoted by $S N(\lambda)$, if it has the probability density function $(p d f)$ below:

$$
f(z)=2 \phi(z) \Phi(\lambda z), \quad z \in R, \lambda \in R,
$$

where $\phi(z)$ and $\Phi(z)$ are the $p d f$ and the cumulative distribution function $(c d f)$ of the standard normal distribution, respectively (Azzalini, 1985). When $\lambda=0, Z$ follows $N(0,1)$. Now, let's extend this to a general $N$ dimensional random vector; that is, an $N \times 1$ random vector $\mathbf{Y}$ has a multivariate $S N$ distribution with location vector $\boldsymbol{\mu} \in R^{N}$, variance matrix $\boldsymbol{\Sigma}$, and skewness vector $\boldsymbol{\lambda} \in R^{N}$, denoted by $\mathbf{Y} \sim S N_{N}(\boldsymbol{\mu}, \boldsymbol{\Sigma}, \boldsymbol{\lambda})$, if its $p d f$ is

$$
f(\mathbf{y})=2 \phi_{N}(\mathbf{y} \mid \boldsymbol{\mu}, \boldsymbol{\Sigma}) \Phi\left(\boldsymbol{\lambda}^{\prime} \boldsymbol{\Sigma}^{-\frac{1}{2}}(\mathbf{y}-\boldsymbol{\mu})\right), \quad \mathbf{y} \in R^{N}
$$

where $\phi_{N}(\cdot)$ is the $p d f$ of the $N(\boldsymbol{\mu}, \boldsymbol{\Sigma})$, (Arellano-Valle et al., 2005). Note that for $\boldsymbol{\lambda}=\mathbf{0}$ the random vector $\mathbf{Y}$ follows $N(\boldsymbol{\mu}, \boldsymbol{\Sigma})$.

To define the $S N I$ distribution, consider an $N \times 1$ random vector $\mathbf{Y}$ with pdf

$$
f(\mathbf{y})=2 \int_{0}^{\infty} \phi_{N}\left(\mathbf{y} \mid \boldsymbol{\mu}, u^{-1} \boldsymbol{\Sigma}\right) \Phi_{1}\left(u^{\frac{1}{2}} \boldsymbol{\lambda}^{\prime} \boldsymbol{\Sigma}^{-\frac{1}{2}}(\mathbf{y}-\boldsymbol{\mu})\right) d H(u),
$$

where $U$ is a positive random variable with cdf $H(u ; \boldsymbol{\nu})$ indexed by the parameter vector $\boldsymbol{\nu}, \boldsymbol{\mu} \in R^{N}$ and $\boldsymbol{\lambda} \in R^{N}$ are location and skewness parameters, respectively, and $\boldsymbol{\Sigma}$ is a positive definite $(p d)$ scale matrix. Then, $\mathbf{Y}$ has an $S N I$ distribution, denoted by $\mathbf{Y} \sim S N I_{N}(\boldsymbol{\mu}, \boldsymbol{\Sigma}, \boldsymbol{\lambda} ; H)$ (Lachos and Labra, 2014). As an example, for the skew-t distribution with $\nu$ degrees of freedom, $S T_{N}(\boldsymbol{\mu}, \boldsymbol{\Sigma}, \boldsymbol{\lambda}, \nu), U \sim \operatorname{Gamma}(\nu / 2, \nu / 2)$, where $\operatorname{Gamma}(\alpha, \beta)$ stands for gamma distribution with mean $\alpha / \beta$. When $\boldsymbol{\lambda}=\mathbf{0}, \mathbf{Y}$ follows an $N I$ distribution. For $\mathbf{Y}$ with $S N I$ distribution, it is also true that for any $\mathbf{a} \in R^{N}$ and $b \in R$

$$
\mathbf{a}^{\prime} \mathbf{Y}+b \sim S N I_{1}\left(\mathbf{a}^{\prime} \boldsymbol{\mu}+b, \mathbf{a}^{\prime} \mathbf{\Sigma} \mathbf{a}, \lambda^{*} ; H\right),
$$

where $\lambda^{*}=\alpha /\left(1-\alpha^{2}\right)^{1 / 2}$ and $\alpha=\left\{\mathbf{a}^{\prime} \boldsymbol{\Sigma} \mathbf{a}\left(1+\boldsymbol{\lambda}^{\prime} \boldsymbol{\lambda}\right)\right\}^{-1 / 2} \mathbf{a}^{\prime} \boldsymbol{\Sigma}^{1 / 2} \boldsymbol{\lambda}$.

We utilize random variables $X_{1}$ and $X_{2}$ with $S N I$ distribution functions $F_{1}$ and $F_{2}$ respectively, and use the following Farlie-Gumbel-Morgenstern 
$(F G M)$ copula function (Balakrishnan and Lai, 2009) with $\alpha \in[-1,1]$, due to the small correlations between $X_{1}$ and $X_{2}$, to come up with joint distribution function $F\left(x_{1}, x_{2}\right)$ as below:

$$
F\left(x_{1}, x_{2}\right)=F_{1}\left(x_{1}\right) F_{2}\left(x_{2}\right)\left\{1+\alpha\left[1-F_{1}\left(x_{1}\right)\right]\left[1-F_{2}\left(x_{2}\right)\right]\right\} .
$$

\section{$3 \quad S P$ Formulization}

It is supposed to investigate the general form of $S P$ for model (2). Following Shirakura et al. (1996) in using their notations for preserving the similarity, yet in a more general context, some formulization may simplify the derivation of $S P$ in the next section.

Let $\mathbf{A}(\boldsymbol{\zeta})=\left[\mathbf{A}_{1}: \mathbf{A}_{21}(\boldsymbol{\zeta})\right]$ and $\boldsymbol{M}(\boldsymbol{\zeta})=\mathbf{A}(\boldsymbol{\zeta})^{\prime} \boldsymbol{\Sigma}_{\boldsymbol{e}}^{-\mathbf{1}} \mathbf{A}(\boldsymbol{\zeta}), S S E$ of model (2) can be written as $s^{2}(\boldsymbol{\zeta})=\mathbf{y}^{\prime} \mathbf{Q}(\boldsymbol{\zeta}) \mathbf{y}$, where $\mathbf{Q}(\boldsymbol{\zeta})=\boldsymbol{\Sigma}_{e}^{-\frac{1}{2}}(\mathbf{I}-\mathbf{P}(\boldsymbol{\zeta})) \boldsymbol{\Sigma}_{e}^{-\frac{1}{2}}$, in which $\boldsymbol{P}(\zeta)=\boldsymbol{\Sigma}_{e}^{-\frac{1}{2}} \mathbf{A}(\zeta) \boldsymbol{M}(\zeta)^{-1} \mathbf{A}(\zeta)^{\prime} \boldsymbol{\Sigma}_{e}^{-\frac{1}{2}}$. By taking $\mathbf{M}=\mathbf{A}_{1}^{\prime} \boldsymbol{\Sigma}_{e}^{-1} \mathbf{A}_{1}$, $\mathbf{P}=\boldsymbol{\Sigma}_{e}^{-\frac{1}{2}} \mathbf{A}_{1} \mathbf{M}^{-1} \mathbf{A}_{1}^{\prime} \boldsymbol{\Sigma}_{e}^{-\frac{1}{2}}, \mathbf{Z}=\boldsymbol{\Sigma}_{e}^{-\frac{1}{2}} \mathbf{Y}$ and $h(\boldsymbol{\zeta}, \mathbf{y})=\mathbf{Z}^{\prime}(\mathbf{P}(\boldsymbol{\zeta})-\mathbf{P}) \mathbf{Z}, s^{2}(\boldsymbol{\zeta})$ can be written as:

$$
s^{2}(\boldsymbol{\zeta})=\mathbf{Z}^{\prime}(\mathbf{I}-\mathbf{P}) \mathbf{Z}-h(\boldsymbol{\zeta}, \mathbf{y}) .
$$

Accordingly, the minimization of $s^{2}(\boldsymbol{\zeta})$ for identifying the true model is equivalent to the maximization of $h(\boldsymbol{\zeta}, \mathbf{y})$ on $\boldsymbol{\zeta}$. For the noiseless case, $\sigma^{2}=0$, $P\left(s^{2}\left(\boldsymbol{\zeta}_{0}\right)<s^{2}(\boldsymbol{\zeta})\right)=P\left(h\left(\boldsymbol{\zeta}_{0}, \mathbf{y}\right)\right)>P(h(\boldsymbol{\zeta}, \mathbf{y}))=1$; however, for $\sigma^{2}>0$ it is less than 1. Shirakura et al. (1996) defined $S P=\min _{\zeta_{0} \in \xi_{2}} \min _{\boldsymbol{\zeta} \neq \boldsymbol{\zeta}_{0}} P\left(h\left(\boldsymbol{\zeta}_{0}, \mathbf{y}\right)>\right.$ $h(\boldsymbol{\zeta}, \mathbf{y}))$, and argued that a larger value of $S P$ is the higher capability of search design in identifying the true model. For $\mathbf{B}=\mathbf{A}_{21}(\boldsymbol{\zeta})^{\prime} \boldsymbol{\Sigma}_{e}^{-\frac{1}{2}}(\mathbf{I}-$ $\mathbf{P}) \boldsymbol{\Sigma}_{e}^{-\frac{1}{2}} \mathbf{A}_{21}(\boldsymbol{\zeta}), h(\boldsymbol{\zeta}, \mathbf{y})$ is:

$$
h(\boldsymbol{\zeta}, \mathbf{y})=\mathbf{Z}^{\prime}(\mathbf{I}-\mathbf{P}) \boldsymbol{\Sigma}_{e}^{-\frac{1}{2}} \mathbf{A}_{21}(\boldsymbol{\zeta}) \mathbf{B}^{-1} \mathbf{A}_{21}(\boldsymbol{\zeta})^{\prime} \boldsymbol{\Sigma}_{e}^{-\frac{1}{2}}(\mathbf{I}-\mathbf{P}) \mathbf{Z} .
$$

Consider the case $k=1$ and suppose $\mathbf{a}(\zeta)$ is the column in $\mathbf{A}_{2}$ corresponding to $\zeta$. By taking $r(\zeta)=\mathbf{a}(\zeta)^{\prime} \boldsymbol{\Sigma}_{e}^{-\frac{1}{2}}(\mathbf{I}-\mathbf{P}) \boldsymbol{\Sigma}_{e}^{-\frac{1}{2}} \mathbf{a}(\zeta)$, the $h(\boldsymbol{\zeta}, \mathbf{y})$ in $(9)$ is expressed as

$$
h(\boldsymbol{\zeta}, \mathbf{y})=\left(\frac{\mathbf{a}(\zeta)^{\prime} \boldsymbol{\Sigma}_{e}^{-\frac{1}{2}}(\mathbf{I}-\mathbf{P}) \mathbf{Z}}{\sqrt{r(\zeta)}}\right)^{2}
$$

By inserting the true model (3) in (10) and from $\boldsymbol{\Sigma}_{e}^{-\frac{1}{2}}(\mathbf{I}-\mathbf{P}) \boldsymbol{\Sigma}_{e}^{-\frac{1}{2}} \mathbf{A}_{1}=\mathbf{0}$, 
we have

$$
\begin{aligned}
h(\boldsymbol{\zeta}, \mathbf{y}) & =\left(\frac{\mathbf{a}(\zeta)^{\prime} \boldsymbol{\Sigma}_{e}^{-\frac{1}{2}}(\mathbf{I}-\mathbf{P}) \boldsymbol{\Sigma}_{e}^{-\frac{1}{2}} \mathbf{a}\left(\zeta_{0}\right)}{\sqrt{r(\zeta)}} \zeta_{0}+\frac{\mathbf{a}(\zeta)^{\prime} \boldsymbol{\Sigma}_{e}^{-\frac{1}{2}}(\mathbf{I}-\mathbf{P}) \mathbf{\Sigma}_{e}^{-\frac{1}{2}}}{\sqrt{r(\zeta)}} \mathbf{e}\right)^{2} \\
& =\left(\mathbf{b}(\zeta)^{\prime} \mathbf{b}\left(\zeta_{0}\right) \sqrt{r\left(\zeta_{0}\right)} \zeta_{0}+\mathbf{b}(\zeta)^{\prime} \boldsymbol{\Sigma}_{e}^{-\frac{1}{2}} \mathbf{e}\right)^{2}
\end{aligned}
$$

in which $\mathbf{b}(\zeta)=(\mathbf{I}-\mathbf{P}) \boldsymbol{\Sigma}_{e}^{-\frac{1}{2}} \mathbf{a}(\zeta) / \sqrt{r(\zeta)}$ and $\mathbf{b}(\zeta)^{\prime} \mathbf{b}(\zeta)=1$.

Now, by $Z=\mathbf{b}(\zeta)^{\prime} \mathbf{b}\left(\zeta_{0}\right) \delta_{0}+\mathbf{b}(\zeta)^{\prime} \boldsymbol{\Sigma}_{e}^{-\frac{1}{2}} \mathbf{e}$, where $\delta_{0}=\sqrt{r\left(\zeta_{0}\right)} \zeta_{0}$, calculation of $Z$ for $\zeta=\zeta_{0}$ gives $Z_{0}=\delta_{0}+\mathbf{b}\left(\zeta_{0}\right)^{\prime} \boldsymbol{\Sigma}_{e}^{-\frac{1}{2}} \mathbf{e}$. For $\zeta \neq \zeta_{0}$,

$$
\begin{aligned}
P\left(h\left(\zeta_{0}, \mathbf{y}\right)>h(\zeta, \mathbf{y})\right) & =P\left(Z_{0}^{2}>Z^{2}\right) \\
& =P\left(\left(Z_{0}+Z\right)\left(Z_{0}-Z\right)>0\right) \\
& =P\left(X_{1}>0, X_{2}>0\right)+P\left(X_{1}<0, X_{2}<0\right),
\end{aligned}
$$

where $X_{1}$ and $X_{2}$ are as below:

$$
\begin{aligned}
& X_{1}=Z_{0}-Z=\left(\mathbf{b}\left(\zeta_{0}\right)-\mathbf{b}(\zeta)\right)^{\prime} \boldsymbol{\Sigma}_{e}^{-\frac{1}{2}} \mathbf{e}+\left(1-\mathbf{b}(\zeta)^{\prime} \mathbf{b}\left(\zeta_{0}\right)\right) \delta_{0}, \\
& X_{2}=Z_{0}+Z=\left(\mathbf{b}\left(\zeta_{0}\right)+\mathbf{b}(\zeta)\right)^{\prime} \boldsymbol{\Sigma}_{e}^{-\frac{1}{2}} \mathbf{e}+\left(1+\mathbf{b}(\zeta)^{\prime} \mathbf{b}\left(\zeta_{0}\right)\right) \delta_{0} .
\end{aligned}
$$

Distributions of $X_{1}$ and $X_{2}$ under the error distributions of model (2) will be given in the next section, while $\left(\mathbf{b}\left(\zeta_{0}\right)-\mathbf{b}(\zeta)\right)^{\prime}\left(\mathbf{b}\left(\zeta_{0}\right)+\mathbf{b}(\zeta)\right)=0$ imply that $X_{1}$ and $X_{2}$ are uncorrelated.

\section{$4 S P$ for $S N / S N I$ Search Model}

Apriority, in model (2) let's assume that $\mathbf{e} \sim S N_{N}\left(\mathbf{0}, \sigma^{2} \boldsymbol{\Sigma}, \boldsymbol{\lambda}\right)$, and let $\boldsymbol{\delta}=$ $\boldsymbol{\lambda} / \sqrt{1+\boldsymbol{\lambda}^{\prime} \boldsymbol{\lambda}}$. Consider the variables $X_{1}$ and $X_{2}$ in (12) and (13), respectively, from (6) distributions of them are easily provided, i.e.,

$$
X_{1} \sim S N\left(\left(1-x\left(\zeta, \zeta_{0}\right)\right) \delta_{0}, \sigma^{2} v\left(\zeta, \zeta_{0}\right), \lambda_{1}^{*}\right)
$$

and

$$
X_{2} \sim S N\left(\left(1+x\left(\zeta, \zeta_{0}\right)\right) \delta_{0}, \sigma^{2} w\left(\zeta, \zeta_{0}\right), \lambda_{2}^{*}\right)
$$


where, $x\left(\zeta, \zeta_{0}\right)=\mathbf{b}(\zeta)^{\prime} \mathbf{b}\left(\zeta_{0}\right), x\left(\zeta, \zeta_{0}\right) \in[-1,1], v\left(\zeta, \zeta_{0}\right)=\mathbf{a}\left(\zeta, \zeta_{0}\right)^{\prime} \mathbf{\Sigma} \mathbf{a}\left(\zeta, \zeta_{0}\right)$, $w\left(\zeta, \zeta_{0}\right)=\mathbf{b}\left(\zeta, \zeta_{0}\right)^{\prime} \mathbf{\Sigma} \mathbf{b}\left(\zeta, \zeta_{0}\right), \mathbf{a}\left(\zeta, \zeta_{0}\right)=\boldsymbol{\Sigma}_{e}^{-\frac{1}{2}}\left(\mathbf{b}\left(\zeta_{0}\right)-\mathbf{b}(\zeta)\right), \mathbf{b}\left(\zeta, \zeta_{0}\right)=$ $\boldsymbol{\Sigma}_{e}^{-\frac{1}{2}}\left(\mathbf{b}\left(\zeta_{0}\right)+\mathbf{b}(\zeta)\right), \lambda_{1}^{*}=\frac{\delta_{1}^{*}}{\left(1-\delta_{1}^{* 2}\right)^{\frac{1}{2}}}, \delta_{1}^{*}=v\left(\zeta, \zeta_{0}\right)^{\frac{-1}{2}} \mathbf{a}\left(\zeta, \zeta_{0}\right)^{\prime} \boldsymbol{\Sigma}^{\frac{1}{2}} \boldsymbol{\delta}, \lambda_{2}^{*}=\frac{\delta_{2}^{*}}{\left(1-\delta_{2}^{* 2}\right)^{\frac{1}{2}}}$, $\delta_{2}^{*}=w\left(\zeta, \zeta_{0}\right)^{\frac{-1}{2}} \mathbf{b}\left(\zeta, \zeta_{0}\right)^{\prime} \boldsymbol{\Sigma}^{\frac{1}{2}} \boldsymbol{\delta}$, and $\boldsymbol{\Sigma}_{e}=\boldsymbol{\Sigma}-\frac{2}{\pi} \boldsymbol{\Sigma}^{\frac{1}{2}} \boldsymbol{\delta} \boldsymbol{\delta}^{\prime} \boldsymbol{\Sigma}^{\frac{1}{2}}$.

Remark 1. For the case in which $\boldsymbol{\Sigma}=\mathbf{I}$ and $\boldsymbol{\lambda}=\lambda \mathbf{j}$, we have $\boldsymbol{\Sigma}_{\mathbf{e}}=$ $\mathbf{I}-2\left(\lambda^{2} / \pi\left(1+N \lambda^{2}\right)\right) \mathbf{j} \mathbf{j}^{\prime}$ and $\boldsymbol{\Sigma}_{\mathbf{e}}^{-1}=\mathbf{I}+h \mathbf{j} \mathbf{j}^{\prime}$, where $\mathbf{j}$ is the $N \times 1$ vector of ones and $h=2 \lambda^{2} /\left\{\pi\left(1+N \lambda^{2}\right)-2 N \lambda^{2}\right\}$.

Now, we provide the $S P$ for the distributional condition of Remark 1 in the following theorem.

Theorem 1. Under $\mathbf{e} \sim S N_{N}\left(\mathbf{0}, \sigma^{2} \boldsymbol{\Sigma}, \boldsymbol{\lambda}\right)$, the $S P$ in (11) is provided and expressed as:

$$
\begin{aligned}
& G\left(x\left(\zeta, \zeta_{0}\right), v\left(\zeta, \zeta_{0}\right), w\left(\zeta, \zeta_{0}\right), \rho, \alpha\right)= \\
& 1-F_{Z_{1}}\left(-\frac{1-x\left(\zeta, \zeta_{0}\right)}{\sqrt{v\left(\zeta, \zeta_{0}\right)}} \sqrt{r\left(\zeta_{0}\right)} \rho\right)-F_{Z_{2}}\left(-\frac{1+x\left(\zeta, \zeta_{0}\right)}{\sqrt{w\left(\zeta, \zeta_{0}\right)}} \sqrt{r\left(\zeta_{0}\right)} \rho\right) \\
& +2 F_{Z_{1}, Z_{2}}\left(-\frac{1-x\left(\zeta, \zeta_{0}\right)}{\sqrt{v\left(\zeta, \zeta_{0}\right)}} \sqrt{r\left(\zeta_{0}\right)} \rho,-\frac{1+x\left(\zeta, \zeta_{0}\right)}{\sqrt{w\left(\zeta, \zeta_{0}\right)}} \sqrt{r\left(\zeta_{0}\right)} \rho\right),
\end{aligned}
$$

where $\rho=\zeta_{0} / \sigma, F_{Z_{1}}$ and $F_{Z_{2}}$, are the cdfs of $S N\left(\lambda_{1}^{*}\right)$ and $S N\left(\lambda_{2}^{*}\right)$, respectively, and $F_{Z_{1}, Z_{2}}$ is the FGM copula given in (7) in which $x_{1}=$ $-\frac{1-x\left(\zeta, \zeta_{0}\right)}{\sqrt{v\left(\zeta, \zeta_{0}\right)}} \sqrt{r\left(\zeta_{0}\right)} \rho$ and $x_{2}=-\frac{1+x\left(\zeta, \zeta_{0}\right)}{\sqrt{w\left(\zeta, \zeta_{0}\right)}} \sqrt{r\left(\zeta_{0}\right)} \rho$.

Proof. Let take $Z_{1}$ and $Z_{2}$ as: $Z_{1}=\frac{X_{1}-\left(1-x\left(\zeta, \zeta_{0}\right)\right) \delta_{0}}{\sigma \sqrt{v\left(\zeta, \zeta_{0}\right)}}$ and $Z_{2}=\frac{X_{2}-\left(1+x\left(\zeta, \zeta_{0}\right)\right) \delta_{0}}{\sigma \sqrt{w\left(\zeta, \zeta_{0}\right)}}$. Clearly, $Z_{1}$ and $Z_{2}$ are distributed as $S N\left(\lambda_{1}^{*}\right)$ and $S N\left(\lambda_{2}^{*}\right)$, respectively. Some calculations give us the following for $S P$ in (11),

$$
\begin{gathered}
1-P\left(Z_{1}<-\frac{1-x\left(\zeta, \zeta_{0}\right)}{\sqrt{v\left(\zeta, \zeta_{0}\right)}} \sqrt{r\left(\zeta_{0}\right)} \rho\right)-P\left(Z_{2}<-\frac{1+x\left(\zeta, \zeta_{0}\right)}{\sqrt{w\left(\zeta, \zeta_{0}\right)}} \sqrt{r\left(\zeta_{0}\right)} \rho\right) \\
+2 P\left(Z_{1}<-\frac{1-x\left(\zeta, \zeta_{0}\right)}{\sqrt{v\left(\zeta, \zeta_{0}\right)}} \sqrt{r\left(\zeta_{0}\right)} \rho, Z_{2}<-\frac{1+x\left(\zeta, \zeta_{0}\right)}{\sqrt{w\left(\zeta, \zeta_{0}\right)}} \sqrt{r\left(\zeta_{0}\right)} \rho\right)
\end{gathered}
$$

which can be easily written as (14), and the proof is complete . 
It should be noted that $G(\cdot)$ function in (14) depends on the parameters $\rho, \boldsymbol{\lambda}$ and $\alpha$ and is also design-dependent. For normal $Z_{1}$ and $Z_{2}$, i.e. $\lambda_{1}^{*}=$ $\lambda_{2}^{*}=0$, the $S P$ in (14) is reduced to a more simple form given in the following corollary.

Corollary 1. If $\delta_{1}^{*}=\delta_{2}^{*}=0$, then $Z_{1}$ and $Z_{2}$ follow $N(0,1)$. Therefore, the $S P$ given in Theorem 1 is simplified as:

$$
\begin{aligned}
G\left(x\left(\zeta, \zeta_{0}\right), v\left(\zeta, \zeta_{0}\right), w\left(\zeta, \zeta_{0}\right), \rho\right)=1 & -\Phi\left(\frac{1-x\left(\zeta, \zeta_{0}\right)}{\sqrt{v\left(\zeta, \zeta_{0}\right)}} \sqrt{r\left(\zeta_{0}\right)} \rho\right) \\
& -\Phi\left(\frac{1+x\left(\zeta, \zeta_{0}\right)}{\sqrt{w\left(\zeta, \zeta_{0}\right)}} \sqrt{r\left(\zeta_{0}\right)} \rho\right) \\
+ & 2 \Phi\left(\frac{1-x\left(\zeta, \zeta_{0}\right)}{\sqrt{v\left(\zeta, \zeta_{0}\right)}} \sqrt{r\left(\zeta_{0}\right)} \rho\right) \\
& \times \Phi\left(\frac{1+x\left(\zeta, \zeta_{0}\right)}{\sqrt{w\left(\zeta, \zeta_{0}\right)}} \sqrt{r\left(\zeta_{0}\right)} \rho\right) .
\end{aligned}
$$

Remark 2. The $G(\cdot)$ function in (15) is symmetric about $\rho$ for $x\left(\zeta, \zeta_{0}\right) \in$ $[-1,1], v\left(\zeta, \zeta_{0}\right)>0, w\left(\zeta, \zeta_{0}\right)>0$, and $G\left(x\left(\zeta, \zeta_{0}\right), v\left(\zeta, \zeta_{0}\right), w\left(\zeta, \zeta_{0}\right), 0\right)=0.5$. Furthermore, $G(\cdot)$ is strictly increasing on $\rho>0$ for fixed $x\left(\zeta, \zeta_{0}\right) \in(-1,1)$, $v\left(\zeta, \zeta_{0}\right)>0$ and $w\left(\zeta, \zeta_{0}\right)>0$.

Remark 3. Although, the expression (15) and the properties given in Remark 2 is very similar to what was obtained by Shirakura et al. (1996), it is different in quiddity due to be obtained under a more general underlying model with $S N$ error.

Remark 4. Three designs $D_{1}, D_{2}$ and $D_{3}$, given in the next section, comprise the condition in Corollary 1. This provides application of (15) on such the designs.

Following Shirakura et al. (1996) in characterizing and deriving the properties of $S P$, the results in the following corollaries will be shown that in addition to some new findings all of the former properties in the above study are preserved, yet under more general present conditions.

Corollary 2. Under the conditions of Remark 1 and Corollary 1, the followings are true.

i) The function $G\left(x\left(\zeta, \zeta_{0}\right), v\left(\zeta, \zeta_{0}\right), w\left(\zeta, \zeta_{0}\right), \rho\right)$ in (15) is symmetric about $\lambda$. 
ii) If $\mathbf{b}(\zeta)^{\prime} \mathbf{j}=0$ and $\mathbf{b}\left(\zeta_{0}\right)^{\prime} \mathbf{j}=0$, then

1. $v\left(\zeta, \zeta_{0}\right)=2\left(1-x\left(\zeta, \zeta_{0}\right)\right)$ and $w\left(\zeta, \zeta_{0}\right)=2\left(1+x\left(\zeta, \zeta_{0}\right)\right)$, and

2. The function $G\left(x\left(\zeta, \zeta_{0}\right), v\left(\zeta, \zeta_{0}\right), w\left(\zeta, \zeta_{0}\right), \rho\right)$ in (15) is reduced to

$$
\begin{aligned}
& \begin{aligned}
G\left(x\left(\zeta, \zeta_{0}\right), d\right)=1 & -\Phi\left(\sqrt{1-x\left(\zeta, \zeta_{0}\right)} d\right)-\Phi\left(\sqrt{1+x\left(\zeta, \zeta_{0}\right)} d\right) \\
& +2 \Phi\left(\sqrt{1-x\left(\zeta, \zeta_{0}\right)} d\right) \Phi\left(\sqrt{1+x\left(\zeta, \zeta_{0}\right)} d\right),
\end{aligned} \\
& \text { where } d=\sqrt{\frac{r\left(\zeta_{0}\right)}{2}} \rho .
\end{aligned}
$$

Corollary 3. The function $G\left(x\left(\zeta, \zeta_{0}\right), d\right)$ in (16) has the following properties:

i) $G\left(x\left(\zeta, \zeta_{0}\right), d\right)=G\left(-x\left(\zeta, \zeta_{0}\right), d\right)$ for $x\left(\zeta, \zeta_{0}\right) \in[-1,1]$.

ii) For a fixed $d \neq 0, G\left(x\left(\zeta, \zeta_{0}\right), d\right)$ is strictly decreasing on $x\left(\zeta, \zeta_{0}\right) \in$ $(-1,1)$.

Now, let's take $\boldsymbol{\Sigma}=\mathbf{I}, \boldsymbol{\lambda}=\lambda \mathbf{j}$ and assume that the conditions of Corollaries 1 and 2(ii) are satisfied. Furthermore, for a fixed $\zeta_{0}$, let $x\left(\zeta_{0}\right)=$ $\max _{\zeta \neq \zeta 0}\left|\mathbf{b}(\zeta)^{\prime} \mathbf{b}\left(\zeta_{0}\right)\right|$. Then, from Corollaries 2 and 3 , the $S P$ can be expressed as

$$
P(\rho, \lambda)=\min _{\zeta_{0}} G\left(x\left(\zeta_{0}\right), \sqrt{\frac{r\left(\zeta_{0}\right)}{2} \rho}\right) .
$$

Remark 2 give $P(\rho, \lambda)=P(-\rho, \lambda)$, thus it can be focused on $P(\rho, \lambda)$ for only $\rho>0$. Furthermore, $P(\rho, \lambda)$ is strictly increasing for $\rho>0$ and $P(\rho, \lambda)>0.5$. The following definition is utilized for ranking $S D$ s based on the designdependent $S P$-criterion.

Definition 1. Suppose $P_{P_{1}}(\rho, \lambda)$ and $P_{P_{2}}(\rho, \lambda)$ are the $S P$ for search designs $P_{1}$ and $P_{2}$, with $\mathrm{N}$ treatments, respectively. Then $P_{1}$ is said to be better than $P_{2}$ for detecting non-zero effect, if $P_{P_{1}}(\rho, \lambda)>P_{P_{2}}(\rho, \lambda)$ for any $\rho>0$. That is, $P_{1}$ identifies the true model (3) with a higher probability than $P_{2}$.

Corollary 4. If $r(\zeta)$ is constant for any $\zeta \in \boldsymbol{\xi}_{2}$, then from Corollary 3(ii) $P(\rho, \lambda)$ in (17), as a function of $\rho$ and $\lambda$, can be written as

$$
P_{D}(\rho, \lambda)=G\left(x^{*}, \sqrt{\frac{r(\zeta)}{2} \rho}\right)
$$


where $x^{*}=\max _{\zeta_{0}} x\left(\zeta_{0}\right)$.

Corollary 4 will be used in the application Section 5 .

Now, suppose that in model (2) $\mathbf{e} \sim S N I_{N}\left(\mathbf{0}, \sigma^{2} \boldsymbol{\Sigma}, \boldsymbol{\lambda} ; H\right)$, then from (6) we have:

$$
X_{1} \sim S N I_{1}\left(\left(1-x\left(\zeta, \zeta_{0}\right)\right) \delta_{0}, \sigma^{2} v\left(\zeta, \zeta_{0}\right), \lambda_{1}^{*} ; H\right)
$$

and

$$
X_{2} \sim S N I_{1}\left(\left(1+x\left(\zeta, \zeta_{0}\right)\right) \delta_{0}, \sigma^{2} w\left(\zeta, \zeta_{0}\right), \lambda_{2}^{*} ; H\right) .
$$

Note that for finite $E\left[U^{-1 / 2}\right]$ and $E\left[U^{-1}\right]$, we have $\boldsymbol{\Sigma}_{\mathbf{e}}=E\left[U^{-1}\right] \boldsymbol{\Sigma}-$ $\frac{2}{\pi} E^{2}\left[U^{-1 / 2}\right] \boldsymbol{\Sigma}^{1 / 2} \boldsymbol{\delta} \boldsymbol{\delta}^{\prime} \boldsymbol{\Sigma}^{1 / 2}$. Under the above scenario, the $S P$ in (11) is the same as what was obtained in (14), in which $F_{Z_{1}}$ and $F_{Z_{2}}$ are the $c d f \mathrm{~s}$ of $S N I_{1}\left(0,1, \lambda_{1}^{*} ; H\right)$ and $S N I_{1}\left(0,1, \lambda_{2}^{*} ; H\right)$, respectively, and hence $F_{Z_{1}, Z_{2}}$ is the $F G M$ copula in (7) based on these $S N I$ marginal distributions.

\section{An Application}

The search model in this section, is assumed to have $S N$ and skew-t error distributions. The adequacy of the proposed $S P$-criterion in comparing search performance of designs is assessed by applying it on three 12-run $S D$ s $D_{1}, D_{2}$ and $D_{3}$, given in Talebi and Esmailzadeh (2015) for a $2^{4}$ factorial experiment. In model (1), let $\boldsymbol{\xi}_{1}$ be the vector of the overall mean and main effects, and $\boldsymbol{\xi}_{2}$ be the vector of 2- and 3-factor interactions and assume $k=1$. Furthermore, assume that 4 -factor interaction is zero. In the following examples the capability of the proposed criterion in ranking the MEP.1's with respect to their search performances is presented. The calculations were done by Matlab and using "sn" package in R software.

Let $\mathbf{e} \sim S N_{12}\left(\mathbf{0}, \sigma^{2} \mathbf{I}, \boldsymbol{\lambda}\right)$. For $\alpha=1 / 6, \rho$ in $[-1,1]$ with increments of 0.1 and $\boldsymbol{\delta}=(0.04,0.17,0.28,0.30,0.35,0.39,0.15,0.22,0.43,0.11,0.40,0.29)^{\prime}$, (14) was used to calculate $S P$, for $D_{1}, D_{2}$ and $D_{3}$. Note that, based on our findings, results are robust to the different values of $\alpha \in[-1,1]$. The results in Tables 1 and 2, show that for all values of $\rho, D_{2}$ is less efficient than both $D_{1}$ and $D_{3}$, while $D_{1}$ is better than $D_{3}$ for $\rho$ in $[-1,-0.5]$ and $[0.4,1]$. For $\rho$ in [-0.4,0.2], which is in the vicinity of zero, $D_{3}$ is better than $D_{1}$. This means $D_{3}$ is superior for small effect sizes.

For the special case $\boldsymbol{\lambda}=\mathbf{j}$, we have $\boldsymbol{\lambda}_{1}^{*}=\boldsymbol{\lambda}_{2}^{*}=0$ and $\mathbf{b}(\zeta)^{\prime} \mathbf{j}=0$ for any $\zeta$ and for all three above designs. Note that, $r(\zeta)$ is not constant for any $\zeta$. We used (17) to calculate the $S P$ s for different values of $\rho$. The results are 
Table 1. $S P$ under $S N$ model for $\rho<0$.

\begin{tabular}{cccccccccccc}
\hline \hline$\rho$ & & -1 & -0.9 & -0.8 & -0.7 & -0.6 & -0.5 & -0.4 & -0.3 & -0.2 & -0.1 \\
\cline { 3 - 10 }$D_{1}$ & 0.9361 & 0.9106 & 0.8770 & 0.8341 & 0.7815 & 0.7211 & 0.6573 & 0.5974 & 0.5496 & 0.5207 \\
$D_{2}$ & 0.9196 & 0.8897 & 0.8519 & 0.8055 & 0.7514 & 0.6902 & 0.6278 & 0.5736 & 0.5341 & 0.5116 \\
$D_{3}$ & 0.9338 & 0.9080 & 0.8745 & 0.8319 & 0.7802 & 0.7208 & 0.6579 & 0.5984 & 0.5508 & 0.5232 \\
\hline
\end{tabular}

Table 2. $S P$ under $S N$ model for $\rho \geqslant 0$.

\begin{tabular}{|c|c|c|c|c|c|c|c|c|c|c|c|}
\hline$\rho$ & 0 & 0.1 & 0.2 & 0.3 & 0.4 & 0.5 & 0.6 & 0.7 & 0.8 & 0.9 & 1 \\
\hline$D_{1}$ & 0.5176 & 0.5201 & 0.5480 & 0.5982 & 0.6622 & 0.7308 & 0.7886 & 0.8381 & 0.8787 & 0.9108 & 0.9356 \\
\hline$D_{2}$ & 0.5096 & 0.5169 & 0.5321 & 0.5695 & 0.6229 & 0.6844 & 0.7465 & 0.8035 & 0.8523 & 0.8918 & 0.9224 \\
\hline$D_{3}$ & 0.5181 & 0.5233 & 0.5515 & 0.5982 & 0.6567 & 0.7204 & 0.7808 & 0.8323 & 0.8746 & 0.9080 & 0.9337 \\
\hline
\end{tabular}

given in Table 3 , for $D_{1}, D_{2}$ and $D_{3}$. It can be seen that for all values of $\rho, D_{1}$ performs better than $D_{2}$ and for $\rho<0.8, D_{1}$ is better than $D_{3}$. For $\rho<0.6 D_{2}$ performs better than $D_{3}$. Then, as $\rho$ gets values greater than 0.5 , $D_{3}$ overcomes $D_{2}$. As $\rho$ tends to 1 , the $S P$ for $D_{1}$ is similar to $D_{3}$ and they are better than $D_{2}$. Such the orders preserve for larger values of $\rho$, although all get closer.

Hereafter, let $\boldsymbol{\xi}_{2}$ is restricted to the set of 2-factor interactions and 3and 4 -factor interactions are all assumed to be zero. Suppose $\boldsymbol{\lambda}=\lambda \mathbf{j}$ and let take $\lambda=1$, then for every 2- factor interaction, say $\zeta, \lambda_{1}^{*}=\boldsymbol{\lambda}_{2}^{*}=0$ and $\mathbf{b}(\zeta)^{\prime} \mathbf{j}=0$, for $D_{1}, D_{2}$ and $D_{3}$. Moreover, for every $\zeta, r(\zeta)=10.67$ for both $D_{1}$ and $D_{3}$ and is 9.333 for $D_{2}$. Therefore, from Corollary 4 we have $P_{D_{1}}(\rho)=P_{D_{2}}(\rho)=G(0.5,5.33 \rho)$ and $P_{D_{2}}(\rho)=G(0.4286,4.66 \rho)$. The above are valid for other values of $\lambda$. Maple software plot command has been run to plot SP against $\rho$ for designs $D_{1}, D_{2}$ and $D_{3}$, and is shown in figure 1 . This plot indicates that $D_{1}$ and $D_{3}$ have the same search ability and they are better than $D_{2}$. Now, for skew-t model let assume $\mathbf{e} \sim S T_{12}\left(\mathbf{0}, \sigma^{2} \mathbf{I}, \boldsymbol{\lambda}, 4\right)$.

Table 3. $S P$ under $S N$ model with $\boldsymbol{\lambda}=\mathbf{j}$, for some values of $\rho$.

\begin{tabular}{|c|c|c|c|c|c|c|c|c|c|c|}
\hline$\rho$ & 0.1 & 0.2 & 0.3 & 0.4 & 0.5 & 0.6 & 0.7 & 0.8 & 0.9 & 1 \\
\hline$D_{1}$ & 0.5144 & 0.5548 & 0.6135 & 0.6805 & 0.7468 & 0.8062 & 0.8557 & 0.8947 & 0.9245 & 0.9467 \\
\hline$D_{2}$ & 0.5132 & 0.5505 & 0.6055 & 0.6698 & 0.7353 & 0.7956 & 0.8471 & 0.8886 & 0.9205 & 0.9444 \\
\hline$D_{3}$ & 0.5126 & 0.5483 & 0.6019 & 0.6661 & 0.7330 & 0.7963 & 0.8515 & 0.8947 & 0.9245 & 0.9467 \\
\hline
\end{tabular}




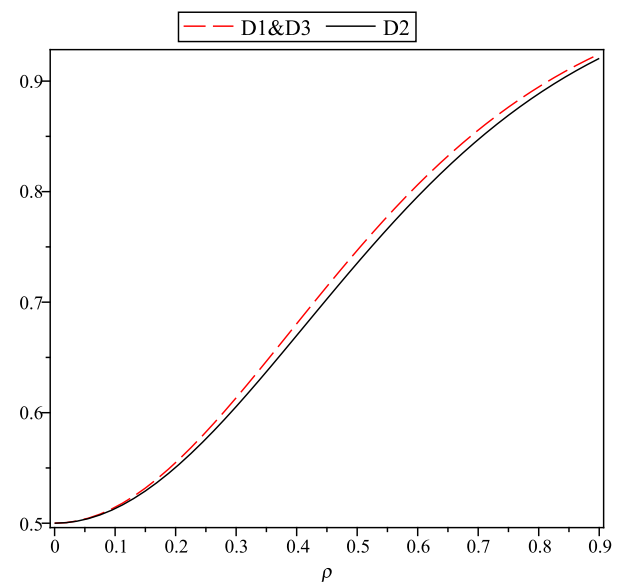

Figure 1. SP versus $\rho$ for $D_{1}, D_{2}$ and $D_{3}$ under SN model with $\boldsymbol{\lambda}=\mathbf{j}$.

Let take $\boldsymbol{\delta}=(0.04,0.17,0.28,0.30,0.35,0.39,0.15,0.22,0.43,0.11,0.40,0.29)^{\prime}$ and $\alpha=1 / 6$. Based on (14) with skew-t marginal distributions, $S P$ s are calculated for different values of $\rho$. The findings for $D_{1}, D_{2}$ and $D_{3}$ are given in Tables 4 and 5 . The results show that the performance behavior depends on $\rho$ values. $D_{3}$ is the superior design for $\rho$ in $[-0.6,0]$ except for $\rho=-0.1$ and $D_{2}$ is less efficient than both $D_{1}$ and $D_{3}$ for values of $\rho$ in [-1,0.8]. It should be noted that for all of the above comparisons the values of criteria are very similar and ranking order of designs is minor, because these 3 designs are highly efficient designs.

\section{Conclusion}

SNI model is more reliable in modeling the real data, due to possible skewness, heavy-tails and correlated error components; moreover, SNI model includes the normal model as a special case. Considering such the more general model, we came up with an intricate and unexplicit distributional format of $S P$-criterion; however, for $S N$ model with $\boldsymbol{\lambda}=\lambda \mathbf{j}$ and subject to some conditions, we derived an explicit $S P$-criterion which is different from $S P$ in Shirakura et al. (1996) in quiddity, but carries all its properties. In application section it was shown that the performance of the new proposed $S P$-criterion depends on the values of components in $\boldsymbol{\lambda}$. This revealed that how ignoring the skewness may cause to misleading values of $S P$ and 
Table 4. $S P$ under $S T$ model for $\rho<0$.

\begin{tabular}{cccccccccccc}
\hline \hline$\rho$ & & -1 & -0.9 & -0.8 & -0.7 & -0.6 & -0.5 & -0.4 & -0.3 & -0.2 & -0.1 \\
\cline { 3 - 10 }$D_{1}$ & 0.8759 & 0.8492 & 0.8170 & 0.7787 & 0.7343 & 0.6850 & 0.6335 & 0.5846 & 0.5454 & 0.5228 \\
$D_{2}$ & 0.8713 & 0.8432 & 0.8092 & 0.7693 & 0.7234 & 0.6732 & 0.6205 & 0.5716 & 0.5359 & 0.5201 \\
$D_{3}$ & 0.8752 & 0.8486 & 0.8166 & 0.7786 & 0.7347 & 0.6859 & 0.6346 & 0.5857 & 0.5458 & 0.5224 \\
\hline
\end{tabular}

Table 5. $S P$ under $S T$ model for $\rho \geqslant 0$.

\begin{tabular}{|c|c|c|c|c|c|c|c|c|c|c|c|}
\hline$\rho$ & 0 & 0.1 & 0.2 & 0.3 & 0.4 & 0.5 & 0.6 & 0.7 & 0.8 & 0.9 & 1 \\
\hline$D_{1}$ & 0.5177 & 0.5247 & 0.5528 & 0.5956 & 0.6458 & 0.6970 & 0.7450 & 0.7876 & 0.8243 & 0.8550 & 0.8805 \\
\hline$D_{2}$ & 0.5103 & 0.5177 & 0.5395 & 0.5772 & 0.6271 & 0.6815 & 0.7343 & 0.7785 & 0.8168 & 0.8492 & 0.8761 \\
\hline$D_{3}$ & 0.5195 & 0.5224 & 0.5464 & 0.5867 & 0.6357 & 0.6870 & 0.7357 & 0.7795 & 0.8173 & 0.8491 & 0.8756 \\
\hline
\end{tabular}

possibly failing in identifying the true model as well as the superior design. Although this votes in favor of the underlying model in this research against the normal model, the $S P$ values could be independent of $\boldsymbol{\lambda}$ for special case $\boldsymbol{\lambda}=\lambda \mathbf{j}$, namely the values of $S P$ are the same as $S P$ for normal model $\lambda=\mathbf{0}$. The condition $\mathbf{b}(\zeta)^{\prime} \mathbf{j}=0$ and $\mathbf{b}\left(\zeta_{0}\right)^{\prime} \mathbf{j}=0$ in Corollary 2(ii) depends on the structure of design, which may lead to some simplification in $S P$. Designs $D_{1}, D_{2}$ and $D_{3}$ which have been taken in Section 4 carry such a structure. That is, the mentioned condition, by which we came up with some simple and explicit form of criterion, is meaningful.

\section{References}

Arellano-Valle, R.B., Bolfarine, H., and Lachos, V.H. (2005). Skew-normal Linear Mixed Models. Journal of Data Science, 3, 415-438.

Arellano-Valle, R.B. and Genton, M.G. (2010). Multivariate Extended Skew-t Distributions and Related Families. Metron, 68, 201-234.

Arnold, B.C., Beaver, R.J., Azzalini, A., Balakrishnan, N., Bhaumik, A., Dey, D.K., Cuadras, C.M. and Sarabia, J.M. (2002). Skewed Multivariate Models Related to Hidden Truncation and/or Selective Reporting. Test, 11, 7-54.

Azzalini, A. (1985). A Class of Distributions which Includes the Normal Ones. Scandinavian journal of Statistics, 12, 171-178.

Bahrami, W. and Qasemi, E. (2015). A Flexible Skew-Generalized Normal Distribution. Journal of Statistical Research of Iran, 11, 131-145. 
Balakrishnan, N. and Lai, C.D. (2009). Continuous Bivariate Distributions, New Zealand, Springer.

Ghosh, S., Shirakura, T. and Srivastava, J.N. (2007). Model Identification Using Search Linear Models and Search Designs. Entropy, Search, Complexity, 85-112.

Ghosh, S. and Teschmacher, L. (2002). Comparisons of Search Designs Using Search Probabilities. Journal of Statistical Planning and Inference, 104, 439-458.

Lachos, V.H. and Labra, F.V. (2014). Multivariate Skew-normal/Independent Distributions: Properties and Inference. Pro Mathematica, 28, 11-53.

Marchenko, Y.V. (2010). Multivariate Skew-t Distributions in Econometrics and Environmetrics, Ph.D thesis, Office of Graduate Studies, Texas A\&M University.

Shirakura, T., Takahashi, T. and Srivastava, J.N. (1996). Searching Probabilities for Nonzero Effects in Search Designs for the Noisy Case. The Annals of Statistics, 24, 2560-2568.

Srivastava, J.N. (1975). Designs for Searching Non-negligible Effects. A Survey of Statistical Design and Linear Models, (ed. J. N. Srivastava), 507-519, North-Holland, Amsterdam.

Talebi, H. and Esmailzadeh, N. (2011a). Using Kullbak-Leibler Distance for Performance Evaluation of Search Designs. Bulletin of the Iranian Mathematical Society, 37, 269-279.

Talebi, H. and Esmailzadeh, N. (2011b). Weighted Searching Probability for Classes of Equivalent Search Designs Comparison. Communications in Statistics-Theory and Methods, 40, 635-647.

Talebi, H. and Esmailzadeh, N. (2015). Comparing Search and Estimation Performances of Designs Based on Compound Criterion. Statistics, 49, 629-637.

\section{Sara Sadeghi}

Department of Statistics, University of Isfahan,

Isfahan, Iran.

email:sarasad205@yahoo.com

\section{Hooshang Talebi}

Department of Statistics, University of Isfahan, Isfahan, Iran.

email: h-talebi@sci.ui.ac.ir 